\title{
THREE EXAMPLES CONCERNING THE ORE CONDITION IN NOETHERIAN RINGS
}

\author{
by A. W. CHATTERS
}

(Received 16th October 1978)

\section{Introduction}

A ring $R$ is said to satisfy the right Ore condition with respect to a subset $C$ of $R$ if, given $a \in R$ and $c \in C$, there exist $b \in R$ and $D \in C$ such that $a d=c b$. It is well known that $R$ has a classical right quotient ring if and only if $R$ satisfies the right Ore condition with respect to $C$ when $C$ is the set of regular elements of $R$ (a regular element of $R$ being an element of $R$ which is not a zero-divisor). It is also well known that not every ring has a classical right quotient ring. If we make the non-trivial assumption that $R$ has a classical right quotient ring, it is natural to ask whether this property also holds in certain rings related to $R$ such as the ring $M_{n}(R)$ of all $n$ by $n$ matrices over $R$. Some answers to this question are known when extra assumptions are made. For example, it was shown by $L$. W. Small in (5) that if $R$ has a classical right quotient ring $Q$ which is right Artinian then $M_{n}(Q)$ is the right quotient ring of $M_{n}(R)$ and $e Q e$ is the right quotient ring of $e R e$ where $e$ is an idempotent element of $R$. Also it was shown by C. R. Hajarnavis in (3) that if $R$ is a Noetherian ring all of whose ideals satisfy the Artin-Rees property then $R$ has a quotient ring $Q$ and $M_{n}(Q)$ is the quotient ring of $M_{n}(R)$.

The first example we give is a right Noetherian ring $R$ which has a right quotient ring but $M_{2}(R)$ does not. The second example is a Noetherian P.I. ring $R$ which is its own quotient ring and which has an idempotent element $e$ such that $e R e$ has neither a right nor a left quotient ring. An ideal $I$ of a ring $R$ is said to satisfy the $A R$-property (short for Artin-Rees property) if, given a right ideal $K$ of $R$, there is a positive integer $n$ such that $K \cap I^{n} \subseteq K I$. The third example we give is a right Noetherian P.I. ring $R$ which has a prime ideal $P$ which satisfies the $A R$-property (in fact is nilpotent) but $R$ does not satisfy the right Ore condition with respect to $C(P)$ where $C(P)$ denotes the set of elements of $R$ whose images are regular elements of $R / P$. In (4) a method is indicated for constructing an example with similar properties, but that method must fail because the ring $R$ which it produces is a principal right ideal ring whose nilpotent radical $P$ is prime; in such a ring $R$ the elements of $C(P)$ are precisely the right regular elements of $R$, and hence $R$ satisfies the right Ore condition with respect to $C(P)$ because of the following result: If $S$ is a right Noetherian ring with nilpotent radical $N$ and if $a, c \in S$ with $c$ right regular, then there exist $b \in S$ and $d \in C(N)$ such that $a d=c b$.

Conventions. $\quad C(0)$ and $C(I)$ will denote respectively the set of regular elements of $R$ and the set of elements of $R$ which are regular $\bmod (I)$; if there is a possibility of ambiguity 
we shall write $C_{R}(0)$ and $C_{R}(I)$. By "the right quotient ring of $R$ " we shall mean the classical right quotient ring of $R$, i.e. the ring formed by inverting all the regular elements of $R$. In constructing matrix rings we shall use the following kind of notation:

$$
\left(\begin{array}{cc}
S & M \\
0 & T
\end{array}\right)=\left\{\left(\begin{array}{cc}
s & m \\
0 & t
\end{array}\right): s \in S, m \in M, t \in T\right\} .
$$

Acknowledgements. I wish to thank the Mathematics Institute of the University of Warwick for its hospitality during the Group Rings and Infinite Groups Symposium. I also wish to thank S. M. Ginn and C. R. Hajarnavis for their stimulating suggestions and encouragement.

\section{The examples}

Example 2.1. We shall show that there is a right Noetherian ring $R$ such that $R$ has a right quotient ring but $M_{2}(R)$ does not. The construction is in several stages and we start by taking $T$ to be any right Noetherian integral domain which is not left Ore (i.e. there are non-zero left ideals $A$ and $B$ of $T$ such that $A \cap B=0$ ). Let $u$ be an indeterminate which commutes with the elements of $T$ and let $C$ denote the set of all elements of the polynomial ring $T[u]$ which have non-zero constant term. We first show that $T[u]$ satisfies the right Ore condition with respect to $C$. We note that $T[u]$ is a right Noetherian domain so that any two non-zero right ideals of $T[u]$ have non-zero intersection. Let $a \in T[u]$ and $c \in C$. For the purposes of establishing the right Ore condition we may suppose that $a \neq 0$. We have $a T[u] \cap c T[u] \neq 0$ so that $a f=c g$ for some non-zero elements $f$ and $g$ of $T[u]$. We can write $f=d u^{i}$ for some $d \in C$ and non-negative-integer $i$. Thus $a d u^{i}=c g$ where $c$ has non-zero constant term. Therefore $g=b u^{i}$ for some $b \in T[u]$. Hence $a d=c b$ with $d \in C$.

Now let $S$ be the partial right quotient ring of $T[u]$ with respect to $C$ and let $D$ be the right quotient division ring of $T$. We can make $D$ into a right $S$-module by identifying $D$ with $S / u S$, i.e. by setting $D u=0$. Set

$$
R=\left(\begin{array}{cc}
T & T \\
0 & T[u]
\end{array}\right) \text { and } Q=\left(\begin{array}{cc}
D & D \\
0 & S
\end{array}\right)
$$

with the usual matrix operations and using the right action of $S$ on $D$ defined above when calculating the $(1,2)$-entry of a product. We shall now show that $Q$ is the right quotient ring of $\boldsymbol{R}$. It is straightforward to check that the regular elements of $R$ are given by

$$
C_{R}(0)=\left\{\left(\begin{array}{ll}
a & b \\
0 & c
\end{array}\right): 0 \neq a \in T, b \in T, c \in C\right\} .
$$

Let

$$
r=\left(\begin{array}{ll}
a & b \\
0 & c
\end{array}\right) \in C_{R}(0)
$$

then $a$ has an inverse $a^{-1}$ in $D, c$ has an inverse $c^{-1}$ in $S$, and

$$
\left(\begin{array}{cc}
a^{-1}, & -a^{-1} b c^{-1} \\
0, & c^{-1}
\end{array}\right)
$$


is an inverse for $r$ in $Q$. Now let

$$
q=\left(\begin{array}{ll}
f & g \\
0 & h
\end{array}\right) \in Q
$$

we must show that $q r \in R$ for some $r \in C_{R}(0)$. Because $D$ is the right quotient ring of $T$ there is a non-zero element $a$ of $T$ such that $f a \in T$ and $g a \in T$. Also there exists $d \in C$ such that $h d \in T[u]$. Because both $a$ and $d$ are elements of $C$ there exists $c \in C$ such that $c \in a T[u] \cap d T[u]$. Set

$$
r=\left(\begin{array}{ll}
a & 0 \\
0 & c
\end{array}\right)
$$

then $r \in C_{R}(0)$ and $q r \in R$. Thus $Q$ is the right quotient ring of $R$. In future we shall omit the details of such verifications.

Finally we shall show that $M_{2}(R)$ does not have a right quotient ring. Because $T$ is not left Ore there are non-zero elements $p$ and $q$ of $T$ such that $T p \cap T q=0$. Thus if $s$ and $t$ are elements of $T$ such that $s p=t q$ then $s=t=0$. Let $x, y, z$ be the following elements of $R$ :

$$
x=\left(\begin{array}{ll}
p & 0 \\
0 & p
\end{array}\right), \quad y=\left(\begin{array}{ll}
q & 0 \\
0 & q
\end{array}\right), \quad \text { and } \quad z=\left(\begin{array}{ll}
1 & 0 \\
0 & u
\end{array}\right) \text {. }
$$

It can easily be shown that $x$ and $y$ are regular elements of $R$ and that $R x \cap R y=0$ (so that $r_{1} x=r_{2} y$ implies $r_{1}=r_{2}=0$ ), and $z$ is right but not left regular (i.e. $z r=0$ implies $r=0$, but there is a non-zero element $r$ of $R$ such that $r z=0$ ). Let $a$ and $c$ be the following elements of $M_{2}(R)$ :

$$
a=\left(\begin{array}{ll}
1 & 0 \\
0 & 0
\end{array}\right) \text { and } c=\left(\begin{array}{ll}
z & x \\
0 & y
\end{array}\right)
$$

It is easy to show that $c$ is a regular element of $M_{2}(R)$ (using $R x \cap R y=0$ to prove left regularity). Suppose that there are elements $b$ and $d$ of $M_{2}(R)$ such that $a d=c b$; we shall show that $d$ is not regular. Let $b=\left[b_{i j}\right]$ and $d=\left[d_{i j}\right]$ with $b_{i j}, d_{i j} \in R$ for $1 \leqq i, j \leqq 2$. Comparing entries in the second row of $a d$ and $c b$ gives $0=y b_{21}=y b_{22}$ so that $b_{21}=b_{22}=$ 0 . Now comparing the first rows of $a d$ and $c b$ gives $d_{11}=z b_{11}$ and $d_{12}=z b_{12}$. But there is a non-zero element $r$ of $R$ such that $r z=0$. Let $w$ be the element of $M_{2}(R)$ with $r$ in the (1, 1)-position and 0's elsewhere, then $w \neq 0$ and $w d=0$. Therefore $d$ is not a regular element of $M_{2}(R)$.

Remarks. (1) It is clear from the last paragraph that the ring of 2 by 2 upper triangular matrices over $R$ does not have a right quotient ring, and therefore neither does the ring of 2 by 2 lower triangular matrices over $R$ because these two rings are conjugate under an inner automorphism of $M_{2}(R)$.

(2) We do not know whether there is a ring $R$ which is its own quotient ring and is such that $M_{2}(R)$ does not have a right quotient ring. Part of the difficulty with this sort of question is the problem of finding a convenient description for the regular elements of $M_{2}(R)$; for example, it is possible for a regular element of $M_{2}(R)$ to have entries which are all zero-divisors in $R$. However, the situation for triangular matrices is more manageable, and $I$ am very grateful to $H$. Attarchi for communicating the following information to me. 
Let $R$ be a ring such that $c R(R c)$ is an essential right (left) ideal of $R$ for each right (left) regular element $c$ of $R$. Suppose that

$$
w=\left(\begin{array}{ll}
a & b \\
0 & c
\end{array}\right)
$$

is a regular element of the ring $U_{2}(R)$ of all 2 by 2 upper triangular matrices over $R$. Clearly $a$ is a right regular element of $R$ and $c$ is left regular. Let $x \in 1(a) \cap R c b^{-1}$ where $1(a)=\{r \in R: r a=0\}$ and $R c b^{-1}=\{r \in R: r b \in R c\}$. There exists $y \in R$ such that $x b=-y c$. Set

$$
z=\left(\begin{array}{ll}
x & y \\
0 & 0
\end{array}\right)
$$

then $z w=0$, so that $z=0$. Thus $1(a) \cap R c b^{-1}=0$ from which it follows that $1(a)=0$ because $R c b^{-1}$ is an essential left ideal of $R$. Thus $a$ is a regular element of $R$, and by symmetry so also is $c$. Thus, for such a ring $R$, a matrix $w$ of the form given above is regular if and only if $a$ and $c$ are regular elements of $R$. This makes it easy to prove that, for example, if $R$ is a left and right Noetherian ring which has a right quotient ring $Q$ then $U_{2}(Q)$ is the right quotient ring of $U_{2}(R)$.

Example 2.2. We shall show that there is a left and right Noetherian P.I. ring $R$ which is its own quotient ring but which has an idempotent element $e$ such that $e R e$ does not have a right or left quotient ring. Again the construction is in several stages. Let $U$ be the ring of integers $Z$ localised at the prime ideal $2 Z$ and set

$$
T=\left(\begin{array}{cc}
U, & 2 U \\
U, & U
\end{array}\right)
$$

then $T$ is a left and right Noetherian prime P.I. ring. Set

$$
M=\left(\begin{array}{cc}
2 U & 2 U \\
U & U
\end{array}\right) \text { and } A=\left(\begin{array}{cc}
2 U & 2 U \\
U & 2 U
\end{array}\right),
$$

then $M$ is a maximal ideal of $T$ and $A$ is an ideal of $T$ (in fact $A$ is the intersection of the two maximal ideals of $T$ ). We have

$$
C_{T}(A)=\left\{\left(\begin{array}{cc}
a & 2 b \\
c & d
\end{array}\right): a, b, c, d \in U, a d \notin 2 U\right\} .
$$

Thus if $t \in C_{T}(A)$ then $\operatorname{det}(t) \in U$ and $\operatorname{det}(t) \notin 2 U$. Thus $\operatorname{det}(t)$ is a unit of $U$, so that the elements of $C_{T}(A)$ are units of $T$.

Set $F=T / M$ then $F \cong Z / 2 Z$. Set

$$
S=\left(\begin{array}{lll}
F & F & F \\
0 & T & F \\
0 & 0 & F
\end{array}\right),
$$

then $S$ is a left and right Noetherian P.I. ring. Let $S^{\prime}$ be the ring which is constructed in the same way as $S$ but using $Z$ instead of $U$, then $S^{\prime}$ is the ring given in (2) and $S$ is a partial 
quotient ring of $S^{\prime}$; this is because $T$ is the partial quotient ring of the ring

$$
T^{\prime}=\left(\begin{array}{cc}
Z & 2 Z \\
Z & Z
\end{array}\right)
$$

with respect to the set of elements of $T^{\prime}$ which have odd determinant. But it was shown in (2) that $S^{\prime}$ has neither a left nor a right quotient ring, and hence $S$ has neither a left nor a right quotient ring; this assertion can be justified either by using the fact that $S$ is a partial quotient ring of $S^{\prime}$, or by modifying the argument given in (2) and using the fact that $T$ does not satisfy either the right or left Ore condition with respect to $C_{T}(M)$.

We now aim to find suitable $R$ and $e$ with $e R e \cong S$. Set

$$
I=\left(\begin{array}{ccc}
0 & F & F \\
0 & A & F \\
0 & 0 & 0
\end{array}\right)
$$

then $I$ is an ideal of $S$. Set $C=C_{S}(0) \cap C_{S}(I)$ and let $s \in S$, then $s \in C$ if and only if $s$ has 1 in the $(1,1)$ - and $(3,3)$-positions and an element of $C_{T}(A)$ in the $(2,2)$-position. It follows easily that the elements of $C$ are units of $S$. Now set

$$
R=\left(\begin{array}{cc}
S & S / I \\
0 & S
\end{array}\right)
$$

then $R$ is a left and right Noetherian P.I. ring. Let $e$ be the element of $R$ which has 1 in the $(1,1)$-position and 0 's elsewhere, then $e$ is idempotent and $e R e \cong S$. An element of $R$ is regular if and only if its diagonal entries belong to $C_{S}(0) \cap C_{S}(I)=C$ (a right or left regular element of $S$ is regular and similarly for the finite commutative ring $S / I$ ). But the elements of $C$ are units of $S$, from which it follows that the regular elements of $R$ are units modulo the nilpotent ideal consisting of all strictly upper triangular elements of $R$. Therefore the regular elements of $R$ are units of $R$.

Example 2.3. We shall show that there is a right Noetherian P.I. ring $R$ which has a prime ideal $P$ such that $P$ satisfies the $A R$-property but $R$ does not satisfy the right Ore condition with respect to $C(P)$. Let $F$ be a field and $x$ an indeterminate. Let $R$ be the ring of all matrices of the form

$$
\left(\begin{array}{cc}
f(0) & g(x) \\
0 & f(x)
\end{array}\right)
$$

with $f(x), g(x) \in F[x]$. Let $t: R \rightarrow F[x]$ be the function which sends the matrix displayed above to $f(x)$ and set $P=\operatorname{Ker}(t)$, then $t$ is a surjective ring homomorphism and $P$ consists of the strictly upper triangular elements of $R$. Thus $P$ is a prime ideal of $R$ and trivially satisfies the $A R$-property because it is nilpotent. Let $e_{i 2}$ denote the element of $R$ with 1 in the (i, 2)-position and 0's elsewhere for $i=1$ or 2. Set $a=e_{12}$ and $c=x e_{22}$ then $c \in C(P)$ and it is not possible to have $a d=c b$ with $b \in R$ and $d \in C(P)$.

Remarks. (1) The following are open questions: (a) If $R$ is a left and right Noetherian ring which has a prime ideal $P$ which satisfies the $A R$-property, does $R$ satisfy the right Ore condition with respect to $C(P)$ ? This question is open even in the special case where $P$ 
is nilpotent. (b) If $R$ is a right Noetherian prime ring which has a non-zero prime ideal $P$ which satisfies the $A R$-property, does $R$ satisfy the right Ore condition with respect to $C(P)$ ?

(2) It is shown in (1), Theorem 3.11, that if $R$ is right Noetherian and $P$ is a prime ideal of $R$ which satisfies the $A R$-property then $R$ satisfies the right Ore condition with respect to $C(P)$ if and only if $P$ is weakly ideal invariant.

\section{REFERENCES}

(1) K. A. Brown, T. H. Lenagan and J. T. Stafford, K-theory and stable structure of Noetherian group rings, University of Warwick (pre-print).

(2) A. W. ChATTERs, A non-singular Noetherian ring need not have a classical quotient ring, $J$. London Math. Soc. (2) 10 (1975), 66-68.

(3) C. R. HAJARNAVIS, Orders in non-commutative quotient rings (Ph.D. thesis, University of Leeds, 1970).

(4) G. O. MICHLER, Right symbolic powers and classical localisation in right Noetherian rings, Math. Z. 127 (1972), 57-69.

(5) L. W. SMALL, Orders in Artinian rings II, J. of Algebra 9 (1968), 266-273.

SCHOOL OF MATHEMATICS

UNIVERSITY WALK

BRISTOL BS8 1TW 